\title{
Relapse of lymphoma in the heart after a 10-year remission
}

\author{
A. J. BELL \\ M.R.C.P.
}

\author{
G. J. MUFTI \\ M.R.C.Path., D.T.M. \& H.
}

\author{
T. J. HAMBLIN \\ M.R.C.P., M.R.C.Path. \\ Royal Victoria Hospital, Boscombe, Bournemouth
}

\begin{abstract}
Summary
A 71-year-old woman presented with intractable cardiac failure $\mathbf{1 0}$ years after receiving treatment for lymphoma.

Extensive investigations failed to demonstrate a recurrence of her disease, but resolution of her cardiac failure following a trial of chemotherapy and subsequently a cross-sectional echocardiogram suggested intracardiac relapse. This was later confirmed at autopsy. Cardiac metastases should be suspected in patients with malignant disease who develop signs or symptoms related to the heart.
\end{abstract}

KEY WORDS: lymphoma, follicular centre cell, centroblastic, diffuse.

\section{Introduction}

Ante-mortem diagnosis of secondary cardiac tumours is made only infrequently and for cardiac involvement to be the sole clinical feature of the disease is extremely rare. Pericardial infiltration is now recognized more frequently, but myocardial or endocardial involvement is usually an autopsy diagnosis. Carcinoma of the breast, carcinoma of the bronchus, malignant melanoma, lymphoma and leukaemia produce cardiac infiltration most commonly and this should be suspected if patients with any of these malignancies develop sudden onset of tachycardias, arrhythmias or congestive cardiac failure, particularly if the latter is resistant to treatment with digoxin and diuretics (Malaret and Aliaga, 1968). A patient in whom late relapse of lymphoma manifested itself as cardiac failure is described.

\section{Case report}

A 71-year-old woman was admitted to hospital in October 1981 with a one-year history of general ill health, increasing shortness of breath, a dry cough, anorexia and weight loss of approximately $10 \mathrm{~kg}$. Ten years previously she had had an excision biopsy of an enlarged lymph node in the left groin and the histology was interpreted as reticulum cell sarcoma. She had been treated with local radiotherapy and was subsequently well, with no evidence of recurrence.

On examination she looked unwell and had a temperature of $37.5^{\circ} \mathrm{C}$. There was no palpable lymphadenopathy or hepatosplenomegaly. The pulse rate was $110 / \mathrm{min}$, regular and the blood pressure was $130 / 80 \mathrm{mmHg}$. The jugular venous pressure was not elevated and no cardiac murmurs or added sounds were audible, although the apex beat was displaced slightly laterally. The remainder of the examination was unremarkable.

The haemoglobin was $10.8 \mathrm{~g} / \mathrm{dl}$ with a MCV of 86 fl and the white cell count was $8.4 \times 10^{9} /$ litre with a normal differential count. The erythrocyte sedimentation rate was $80 \mathrm{~mm}$ in the lst hr. Urea and electrolytes, serum thyroxine, serum protein electrophoresis and liver function tests were all normal and blood and urine cultures were sterile. The chest roentgenogram showed a moderately enlarged heart and clear lung fields and an electrocardiogram showed a sinus tachycardia with very low voltage complexes and a normal axis. An initial diagnosis of idiopathic cardiomyopathy was made.

Two weeks after admission she developed signs of rapidly worsening biventricular failure. The pulse rate rose to $130 / \mathrm{min}$ and the blood pressure fell to $90 / 60 \mathrm{mmHg}$, with poor peripheral perfusion and oliguria. The liver enlarged to $4 \mathrm{~cm}$ below the costal margin and was non-pulsatile and non-tender. The central venous pressure, which was originally +4.5 $\mathrm{cm}$ water rose rapidly to $+13 \mathrm{~cm}$ water and treatment with digoxin and frusemide produced little improvement. In view of her rapid deterioration and past history of lymphoma, she was treated with COP chemotherapy (cyclophosphamide $1 \mathrm{~g}$ i.v.; vincristine $2 \mathrm{mg}$ i.v.; prednisolone $40 \mathrm{mg}$ daily for 5 days). A rapid and dramatic improvement in her clinical state 
ensued, the central venous pressure falling to normal and the blood pressure rising to $140 / 80$ $\mathrm{mmHg}$.

In November 1981 she was well and clinical and radiographic examination, including a lymphangiogram, was normal. A liver biopsy showed dilatation of centrizonal sinusoids compatible with congestive cardiac failure, but no evidence of lymphoma. A bone marrow trephine biopsy was normal and surface marker studies on peripheral blood and marrow lymphocytes failed to reveal a monoclonal population. In December 1981 she was readmitted as an emergency with a 2-day history of palpitations, shortness of breath and a dry cough. On examination she was cachectic and there were signs of bilateral pleural effusions. The jugular venous pressure was elevated to $4 \mathrm{~cm}$ above the sternal angle and there was a sinus tachycardia of $140 / \mathrm{min}$. On this occasion a faint tricuspid diastolic murmur was audible. A chest roentgenogram confirmed the presence of bilateral pleural effusions and again showed cardiomegaly. Microscopy of the pleural effusions and again showed cardiomegaly. Microscopy of the pleural fluid showed normal macrophages and scanty lymphocytes only and cultures were sterile. Crosssectional echocardiography was performed and showed a moderately large tumour mass arising from the free right atrial wall and also a hypokinetic right ventricle (Fig. 1). A second course of COP chemotherapy again produced prompt clinical improvement, but a month later signs of cardiac decompensation reappeared and the patient died.

Autopsy examination revealed a massively enlarged heart (weight $0.65 \mathrm{~kg}$ ) in which the right atrium and right ventricle were extensively replaced with tumour. A tumour mass was protruding into the cavity of the right atrium. Tumour deposits were also present on the parietal pleura and retrosternally but none was found below the diaphragm.

The histological appearances were interpreted as those of follicular centre cell lymphoma, centroblastic, diffuse, (Kiel classification) (Gerard-Marchant $\boldsymbol{e t}$ al., 1974). Comparison of the sections with those obtained 10 years earlier showed identical appearances.

\section{Discussion}

Lymphoma is one of the commonest tumours to metastasize to the heart and Roberts, Glancy and DeVita $\cdot(1968)$ in a series of 196 patients with lymphoma, found evidence of cardiac metastases at autopsy in $24 \%$. Tumour deposits were observed on gross examination of the heart in slightly more than half of the cases; in the remainder lymphoma was detected only after study of histological sections. The incidence of dyspnoea, chest pain, effusions into body cavities, precordial murmurs, ventricular gale lops, oedema and electrocardiographic disturbances was similar in patients with and without cardiac. lymphoma and in only $10 \%$ of cases could clinicas features of cardiac dysfunction during life be attributed to lymphomatous involvement of the heart.

Symptoms and signs resulting from cardiac infil ${ }_{0}^{\Phi}$ tration are, of course, related to the layer of the heart involved. Pericardial infiltration is recognized most? frequently and produces chest pain, pericardia $P$ effusions with or without tamponade, constrictive $\overrightarrow{\vec{H}}$ pericarditis and arrhythmias. Myocardial and endos cardial deposits tend to be more occult but mas produce dysrhythmias, including heart block, embo 3 . lism or obstruction (Roberts et al., 1968). Lymphomai in the right atrium causing tricuspid valve obstruc tion and right heart failure has been reported occasionally, but is rare (Olsen, Bach-Nielsen and Piper, 1962; Garfein, 1976). In the patient described, the diagnosis was made particularly difficult by the absence of detectable disease elsewhere and by the long disease-free interval. A striking response to chemotherapy supported the presumptive diagnosis and further evidence was provided by cross-sectiona echocardiography. Angiography and endomyocare dial biopsy are also useful diagnostic techniques, ghe latter being especially valuable in excluding at ado myxoma which demands surgical treatment (Johnख and Popple, 1980).

Diagnosis of cardiac metastases in patients witl? lymphoma is important because radiation therapy may produce useful palliation. In one series, over hale of the patients so treated responded and some live free of cardiac symptoms, for more than a yea? (Cham et al., 1975). Terry and Kligerman (1970 reported 7 patients with cardiac involvement from lymphomas and leukaemias who were treated with radiotherapy. Two patients (both with Hodgkin's disease involving the pericardium) were alive and apparently disease-free at 2 years and year, respectively, and 3 of the remaining patients achieved excellent palliation. Response of cardiac lymphoma to chemotherapy has also been documented (Garfein, 1976; Conde et al., 1975). This was clearly the case in our patient, and the mecha nism was probably relief of tricuspid valve obstruc $\frac{}{5}$ tion.

However, although the clinical response to chemoos therapy was gratifyingly prompt and dramatic, the patient died 1 month after her second course of treatment. The outcome of this case is similiar to tha of two previously reported patients with non? Hodgkin's lymphoma involving the heart; both ha\& short survivals despite well-documented initial re sponses to chemotherapy (Garfein, 1976; Conde et al., 1975). 
Clinical reports
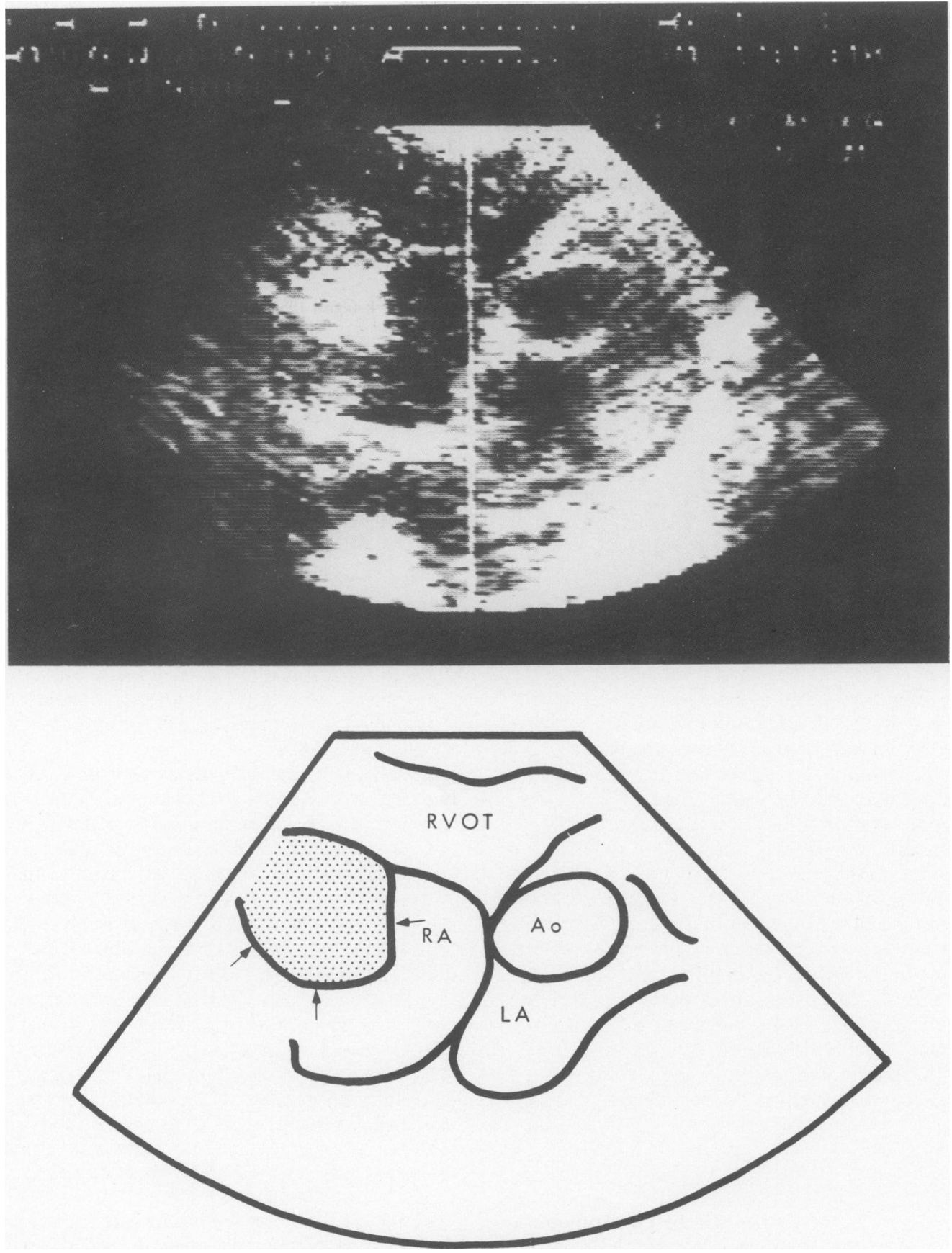

FIG. 1. Cross-sectional echocardiogram showing tumour mass (arrowed) in right atrium (RA). RVOT=right ventricular outflow tract; $A O=$ aorta LA $=$ left atrium

\section{Acknowledgment}

We are grateful to Miss Talbot for typing the manuscript.

\section{References}

Cham, W.C., Freiman, A.H., Carstens, P.H.B. \& Chu, F.C.H., 
(1975) Radiation therapy of cardiac and pericardial metastases. Radiology, 114, 701.

Conde, C.A., Meller, J., Pantaryopoulos, J., Donoso, E. \& DACK, S. (1975) Reticulum cell sarcoma with intracardiac metastases. American Journal of Cardiology, 36, 357.

GaRfEIN, O.B. (1976) Lymphosarcoma of the right atrium. Archives of Internal Medicine, 135, 325.

Gerard-Marchant, R., Hamlin, I., Lennert, K., Rilke, F., STANSFELD, A.G. \& VAN UNNIK, J.A.M. (1974) Classification of non-Hodgkin's lymphomas. Lancet, ii, 406.

Johnson, I.D.A. \& POPPLE, A.W. (1980) Right ventricular outflow tract obstruction secondary to small intestinal lymphoma. British Heart Journal, 43, 593.

MALARET, G.E. \& AliagA, P. (1968) Metastatic disease to the heart. Cancer, 22, 457.
Olsen, S., Bach-Nielsen, P. \& PiPer, J. (1962) Polypoid tumour of the cardiac auricles. Acta Medica Scandinavia, 171, 637.

RoberTs, W.C., GlaNCY, D.L. \& DEVITA, V.T. Jr (1968) Heart in. malignant lymphoma (Hodgkin's disease, lymphosarcoma, reticu lum cell sarcoma and mycosis fungoides). American Journal of Cardiology, 22, 85 .

TerRy, L.N. \& Kligerman, M.M. (1970) Pericardial and myocar dial involvement by lymphomas and leukaemias. The role of radiotherapy. Cancer, 25, 1003.

(Accepted 4 August 1983) 